\title{
Mating with immunised male mice affects the phenotype of adult progeny
}

\author{
Ludmila Gerlinskaya', Margarita Anisimova ${ }^{1}$, Galina Kontsevaya ${ }^{1}$, Svetlana Maslennikova ${ }^{1}$, \\ Alexander Romashchenko ${ }^{1,2}$, Yanli Gong ${ }^{1}$, Yuri Moshkin ${ }^{1}$ and Mikhail Moshkin ${ }^{1,3}$ \\ ${ }^{1}$ Federal Research Center Institute of Cytology and Genetics, Siberian Branch of RAS, Novosibirsk, Russia, \\ ${ }^{2}$ International Tomography Center, Siberian Branch of RAS, Novosibirsk, Russia and ${ }^{3}$ Tomsk State University, \\ Tomsk, Russia \\ Correspondence should be addressed to L Gerlinskaya or M Moshkin; Email: Igerlinskaya@gmail.com ormmp@bionet.nsc.ru
}

\begin{abstract}
The life-history theory suggests that parental experience of the environment is passed to offspring, which allows them to adapt to prevailing conditions. This idea is supported from the mother's side, but to a much less extent from the father's side. Here, we investigated the effect of immunising fathers on pre- and neonatal development and on immune and neuroendocrine phenotypes of their offspring in $\mathrm{C} 57 \mathrm{BL} / 6 \mathrm{~J}$ mice. Nine days before mating, fathers were intraperitoneally injected with the immunogenic protein keyhole limpet hemocyanin (KLH). Females mated with immunised males had less pre-weaning mortality of newborns compared to those mated with control males. Although the antibody response to KLH was similar for the male offspring of control and immunised fathers, the mass indexes of their main immune organs and their androgen response differed significantly. The mass indexes of the thymus and spleen in adult male offspring of immunised fathers were higher compared with the control offspring. The plasma testosterone levels were significantly decreased after KLH administration in the male offspring of control but not of immunised fathers. This was correlated with changes in sperm average path and straight-line velocities. Finally, excitatory neurotransmitters prevailed over inhibitory ones in the amygdala of the progeny of immunised fathers, while in control offspring, the opposite occurred. This is indicative of complex behavioural changes in the offspring of immunised fathers, including sexual ones. Therefore, the paternal experience of foreign antigens modulates the immune and neuroendocrine systems of their progeny, suggesting possible survival and reproductive adaptations to parasitic pressure.

Reproduction (2020) $\mathbf{1 6 0} 117-127$
\end{abstract}

\section{Introduction}

According to the life-history theory, host populations can respond to parasitic pressure by increasing their reproduction effort (Stearns 1992, McNamara \& Houston 1996, Luu \& Tate 2017). Effective reproduction under these conditions requires the preservation of reproductive potential despite the activation of innate and adaptive immunity. However, the exposure of males to non-replicating antigens, mimicking pathogenmediated immune-neuroendocrine responses, leads to a decrease in their sexual attractiveness (Moshkin et al. 2002, Faivre et al. 2003, Zala et al. 2004, Arakawa et al. 2010). At the same time, this may only hamper the reproductive output of immunised males when a free choice of mating partner exists. However, in natural environments, the mating choice is constrained by a social organisation and in the laboratory by experimental design (Potts et al. 1991).

We previously found that despite a decrease in odour attractiveness and sexual activity, immunised male mice showed a greater reproductive output when coupled with intact females who had no mating choice (Gerlinskaya et al. 2012), which resulted in an increased embryo growth rate (Gerlinskaya et al. 2017). In other words, the signalling and behavioural effects of antigenic stimuli do not diminish the male's reproductive abilities per se, and they can be fully realised in the absence of free mating choice. Therefore, as mating by antigen-stimulated males is expected to prevail in host populations living under parasitic pressure, we wondered if this paternal experience and immune-reproductive benefits can be passed on to the offspring.

Indeed, transgenerational transmission of paternal experiences, either beneficial or not, has already been reported in several studies. For instance, the offspring of male mice who experienced defeats in the repeated social conflicts prior to mating acquired anxiety and depressive behaviours (Dietz et al. 2011). Similarly, paternal olfactory fear conditioning with acetophenone strengthened the startle response in their offspring (Dias \& Ressler 2014). Also, in mice whose fathers 
were stressed before mating, a smaller adrenocortical response to immobilisation compared with the progeny of control fathers was observed (Rodgers et al. 2013, 2015).

In the present work, we coupled intact females with antigen-treated and non-treated (control) males to study the mating rate, prenatal development, postnatal survival, and phenotypic characteristics of adult offspring. The immunogenic protein keyhole limpet hemocyanin $(\mathrm{KLH})$ was used as an antigenic stimulus in both fathers and offspring. The main principle of addressing the transgenerational transmission of parental experience consists of evaluating the adaptation and response of offspring to the same stimuli that their parents experienced prior to mating. Following this, we analysed the progeny of antigen-stimulated males for their immune response to the KLH injection and the mass indexes of the main immunocompetent organs, including the thymus and spleen. From the perspective of the life-history theory, the immune traits might tradeoff with the reproductive traits in hosts under parasitic pressure (Luu \& Tate 2017). Thus, we investigated the changes in plasma testosterone and spermatogenesis after $\mathrm{KLH}$ administration in the male offspring of control and $\mathrm{KLH}$-immunised fathers. Using magnetic resonance spectroscopy (MRS), we also studied the neurometabolic profiles of the amygdala and cortex in both offspring. The amygdala is a critical regulatory site for the control of male sexual behaviour (Dominguez et al. 2001, Gresham et al. 2016), which is modulated by a balance between excitatory (glutamate/glutamine-GLX) and inhibitory (GABA) neurotransmitters (Tzanoulinou et al. 2014, Adams \& Rosenkranz 2016). Both of them can be detected in vivo by MRS (Moshkin et al. 2014). Overall, the immunisation of males with the foreign antigen 9-15 days before mating significantly affected the brain, immune and reproductive traits of their sons.

\section{Materials and methods}

\section{Animals and procedures}

Specific pathogen-free (SPF) C57BL mice 12-14 weeks of age were used in this study (55 males and 60 females). All animals and experiments were handled and performed in accordance with the regulations and guidelines of the Animal Care and Use Committee of the Federal Research Centre Institute of Cytology and Genetics operating under standards set by the Federal Health Ministry (2010/708n/RF), NRC, and FELASA. Mice were maintained in a controlled environment with the following conditions: a photoperiod of $14 \mathrm{~h}$ light:10 h dark, a temperature of $22-24^{\circ} \mathrm{C}$, and a humidity of $40-50 \%$. Food (SNIFF, Germany) and water were given ad libitum. To induce an immune response, males were intraperitoneally injected with the immunogenic protein KLH (Native, Sigma, SRP6195) in dose $(50 \mu \mathrm{g}$ per male in $100 \mu \mathrm{L}$ saline).

\section{Experimental groups}

(1) Twenty-five males were used to study immune and endocrine responses to $\mathrm{KLH}$. The dynamics of the immune response were studied in five immunised males. Blood samples for anti-KLH IgG assayed were taken from the retroorbital sinus 3, 9, 15, and 21 days after the immunisation.

Immune and endocrine characteristics before mating were assessed in ten immunised and ten control males. Nine days after the injections of $\mathrm{KLH}$ or saline, males were euthanised by decapitation. Trunk blood was collected and centrifuged at $1000 \mathrm{~g}$ for $15 \mathrm{~min}$. Blood plasma was stored at $-80^{\circ} \mathrm{C}$ until use. Seminal plasma was sampled by gentle massaging of the seminal vesicles, immediately diluted with $500 \mu \mathrm{L}$ of sterile PBS, and centrifuged for $10 \mathrm{~min}$ at $10000 \mathrm{~g}$. The supernatant was collected and stored at $-80^{\circ} \mathrm{C}$ until use.

(2) In breeding experiments, males were divided into two equal groups. Experimental animals were injected with $\mathrm{KLH}$ (KLH group, $n=15)$, and control animals were injected with saline (control group, $n=15$ ). Nine days after the injection, two virgin females were placed with each male immediately after turning the lights off. In total, 60 females were randomly distributed between the control and immunised males and housed with them for 6 days, a time that is sufficient to reach oestrous by every female of the C57BL/6) strain (Nelson et al. 1982, Byers et al. 2012). In this way, the number of females in each experimental group (30) should minimise any effect of asynchrony in their oestrous cycles. The next morning and for the following 6 days, the females were examined daily for the presence of a vaginal plug. The morning on which a vaginal plug was detected was designated day 1 of pregnancy. Upon the detection of mated females, they were moved to separate cages. Six days after coupling (15 days after the injection of KLH or saline), immunised and control males were euthanised by decapitation. Blood plasma, seminal fluids, and epididymis were collected. Pregnancies were detected in 21 out of 30 females in the control group and 25 out of 30 females in the $\mathrm{KLH}$ group. The successful pregnancies were scored either by an autopsy of females on day 16 after the detection of a vaginal plug or by pup delivery.

(3) Pregnant females were separated into two subgroups. Females from the first subgroup (12 from the control group and 15 from the KLH group) were euthanised by decapitation on the 16 th day of pregnancy. Blood plasma was collected and stored at $-80^{\circ} \mathrm{C}$ until use. The pregnant females were autopsied, and the amniotic membranes were cut to accurately collect the amniotic fluid into a tube, which was stored at $-80^{\circ} \mathrm{C}$ until use. The fetuses and placentas were separated and weighed. The remaining pregnant females (nine from the control group and ten from the KLH group) delivered and fed their offspring. During the feeding period, daily inspections of the litters were performed.

(4) Three-week-old offspring were removed from their mothers and kept by unisex groups, with no more than five animals per cage. All offspring were weighed at the age of 3 weeks. At 12-14 weeks of age, male offspring were used for magnetic resonance spectroscopy (MRS), as well as immune and endocrine studies. 


\section{Magnetic resonance spectroscopy}

Brain metabolites were studied in the male offspring from control $(n=5)$ and immunised fathers $(n=5)$. Metabolite concentrations were determined by MRS. A horizontal tomography with a magnetic field of 11.7 T (Bruker, Biospec 117/16 USR, Germany) and a 9-cm inner-diameter gradient (amplitude: 750 $\mathrm{mT} / \mathrm{m}$ and slew rate: $6660 \mathrm{~T} / \mathrm{m} / \mathrm{s}$ ) were used. The determined metabolites and their concentrations were comparable with the data of HPLC and NMR of brain homogenates (Zieminska et al. 2018). Radiofrequency transmission and reception were achieved with transmitter volume $(500.3 \mathrm{MHz}$; distribution $72 / 89 \mathrm{~mm})$ and receiver quadrature surface $(500.3 \mathrm{MHz}$; $123 \times 64 \times 31 \mathrm{~mm}$ ) ${ }^{1} \mathrm{H}$ radiofrequency coils (Bruker, Biospec, Germany). All proton spectra of the amygdala and cortex were recorded (see Moshkin et al. 2014 for a detailed description).

\section{Immunisation of adult offspring}

The offspring males from control $(n=14)$ and antigen-treated fathers $(n=14)$ were divided into two equal groups. KLH (50 $\mu \mathrm{g}$ per male in $100 \mu \mathrm{L}$ saline) and saline (100 $\mu \mathrm{L}$ per male) were intraperitoneal injected. Nine days after the KLH or saline injections, animals were weighed, killed by decapitation, and the thymus, spleen, testicles, preputial glands, seminal vesicles, and epididymis were removed and weighed. Trunk blood was collected and centrifuged at $3000 \mathrm{rpm}$ for $15 \mathrm{~min}$. Blood plasma was stored at $-80^{\circ} \mathrm{C}$ until use.

\section{Determination of the $K L H$-specific immunoglobulin $G(\lg G)$}

The plasma and seminal fluid content of anti-KLH IgG were determined using an ELISA. The plasma of saline-treated males was used as a negative control. ELISA plates were coated with $\mathrm{KLH}(50 \mu \mathrm{L})$ at a concentration of $10 \mathrm{mg} / \mathrm{mL}$ in phosphate buffered saline (PBS), incubated overnight at $4^{\circ} \mathrm{C}$. Plates were washed twice with PBS containing 0.01\% Tween-20 (PBS-T) and blocked with $1 \%$ BSA in PBS-T at room temperature for $2 \mathrm{~h}$ and washed with PBS-T. The anti-KLH IgG level was measured in diluted 1:200 blood plasma and undiluted seminal fluid samples. Blood plasma or seminal fluid $(100 \mu \mathrm{L})$ was added to the $\mathrm{KLH}$-coated wells. Plates were incubated for $3 \mathrm{~h}$ at $37^{\circ} \mathrm{C}$ and washed with PBS-T. Secondary biotinylated anti-mouse $\operatorname{lgG}$ antibodies (100 $\mu \mathrm{L}, 1: 10000$, BD Biosciences) were added and incubated for $1 \mathrm{~h}$ at $37^{\circ} \mathrm{C}$. The plates were then washed, and $100 \mu \mathrm{L}$ of the conjugate (streptavidin-peroxidase 1:5000) was added. After incubating for $1 \mathrm{~h}$ at $37^{\circ} \mathrm{C}$ and washing, $100 \mu \mathrm{L}$ of the ABTS substrate (Sigma-Aldrich) was added and incubated at room temperature for $30 \mathrm{~min}$. The colour reaction was stopped with $10 \% \mathrm{H}_{2} \mathrm{SO}_{4}$. The $\mathrm{OD}_{450}$ (optical density) was measured using a plate reader (iMarkTM, Bio-RAD). The $\mathrm{OD}_{450}$ values of the plasma and seminal fluid of saline-treated males did not exceed the blank values.

\section{Characteristics of spermatozoa}

For the analysis of spermatozoa, the cauda of the epididymis was transferred into tubes containing $500 \mu \mathrm{L}$ of prewarmed $\left(37^{\circ} \mathrm{C}\right.$ ) Hanks solution (Sigma) without $\mathrm{Mg}^{2+}$ or $\mathrm{Ca}^{2+}$. Five to six sectional cuts were made, and the samples were incubated for $20 \mathrm{~min}$ at $37^{\circ} \mathrm{C}$. Spermatozoa concentration, $10^{6} \mathrm{sperm} /$ $\mathrm{mL}$; size $\left(\mu \mathrm{m}^{2}\right)$; mean head size $\left(\mu \mathrm{m}^{2}\right)$; elongation sperm head (\% of head width to head length); motility (\% of total sperm moving at path velocity $\geq 30 \mu \mathrm{m} / \mathrm{s}$ and progressive velocity $\geq 15 \mu \mathrm{m} / \mathrm{s}) ; \mathrm{VAP}$, average-path velocity $(\mu \mathrm{m} / \mathrm{s}) ; \mathrm{VSL}$, straightline velocity $(\mu \mathrm{m} / \mathrm{s}) ; \mathrm{VCL}$, curvilinear velocity $(\mu \mathrm{m} / \mathrm{s}) ; \mathrm{BSF}$, beat cross frequency $(\mathrm{Hz}) ; \mathrm{STR}$, straightness $(\%, \mathrm{VSL} / \mathrm{VAP})$; and $\mathrm{LIN}$, linearity (\%, VSL/CL) were measured using an automatic Mouse-Traxx semen analyser (Hamilton Thorne, USA). In each sample, five fields ( $20 \mu \mathrm{m}$ deep Micro Cell Counting chamber) were examined at $\times 4$ magnification.

\section{Immunoassay}

An ELISA was used to determine the concentration of hormones and cytokines in blood plasma, amniotic fluids, and seminal fluids. Measurements were conducted according to the manufacturer's instructions and as previously described (Gerlinskaya et al. 2017). The minimal testosterone concentration, which could be reliably detected, was $0.087 \mathrm{ng} /$ $\mathrm{mL}$, and the intra- and inter-assay coefficients of variations (CVs) were 8.2 and $5.6 \%$, respectively (Testosterone-IFA Kit, HEMA Ltd.). The minimal detectable progesterone concentration was $0.15 \mathrm{ng} / \mathrm{mL}$, and the intra- and inter-assay CVs were 7.6 and $4.3 \%$, respectively (Progesterone-IFA Kit, HEMA Ltd.). Granulocyte-macrophage colony-stimulating factor (GM-CSF) concentrations were measured in the amniotic fluid using a mouse GM-CSF ELISA Kit (BD Bioscience). The intra- and interassay CVs were 5.2 and $3.9 \%$, respectively. Blood GM-CSF levels were below the detection limit. Tumour necrosis factor (TNF) concentrations were measured in the seminal fluid using a mouse TNF-alpha ELISA Kit (eBioscience, USA). The intraand inter-assay CVs were 7.4 and 9.5\%, respectively.

\section{Statistical analysis}

Following the Kolmogorov-Smirnov test, parametric or nonparametric statistics were used. To elucidate the statistical significance of the paternal effect and effects of offspring immunisation, parametric and non-parametric (Friedman test) two-way ANOVA (ANOVA) tests were used. To analyse the data obtained from the offspring, each individual was treated as a separate unit. Paternal effects on offspring body mass were analysed by the analysis of covariance (ANCOVA) with the immunisation of the father and sex of offspring as the factors and litter size before weaning as the covariate. We used the Student's $t$-test to compare means of the normally distributed traits. The non-parametric Mann-Whitney $U$ test was conducted for plasma and amniotic testosterone, $\lg G$ in blood and seminal plasma, and TNF in the seminal fluid to evaluate the statistical differences between groups. Pearson's correlation coefficients $(r)$ were calculated for spermatozoa characteristics and plasma testosterone. The difference between the sum of progeny delivered by the females mated with the control and KLH-treated males was assessed by the chi-squared $\left(\chi^{2}\right)$ test. Statistical significance was considered at $P<0.05$. Data are presented as the mean \pm S.E.M.(S.E.). 


\section{Results}

\section{Immune and endocrine responses to $K L H$ in male mice (fathers)}

The plasma concentration of anti-KLH IgG in isolated males was significantly increased on day 9 after the intraperitoneal injection of $\mathrm{KLH}$, and then it remained unchanged up to day 21 (Fig. 1A). This suggests that males coupled with intact females between days 9 and 15 after the immunisation were in the steady state of antibody formation. The $\mathrm{OD}_{450}$ values reflecting the levels of anti-KLH IgG in the blood and seminal plasma did not differ in immunised males before and after mating (Fig. 1B). The concentrations of antiKLH IgG were positively correlated between the blood and seminal plasma $(r=0.54, P<0.005, n=25)$.

A non-parametric two-way ANOVA (Friedman test) revealed statistically significant effects of the immunisation $\left(F_{1,46}=4.21, P=0.046\right)$, mating $\left(F_{1,46}=5.20, P=0.027\right)$, and of the interaction between these two factors $\left(F_{1,46}=13.30, P<0.001\right)$ on plasma testosterone (Fig. 1C). Before mating (9 days after the immunisation), plasma testosterone was slightly, but insignificantly, lower in the immunised fathers compared with the control. After mating (15 days after the immunisation), the immunised males showed higher plasma testosterone in comparison with the
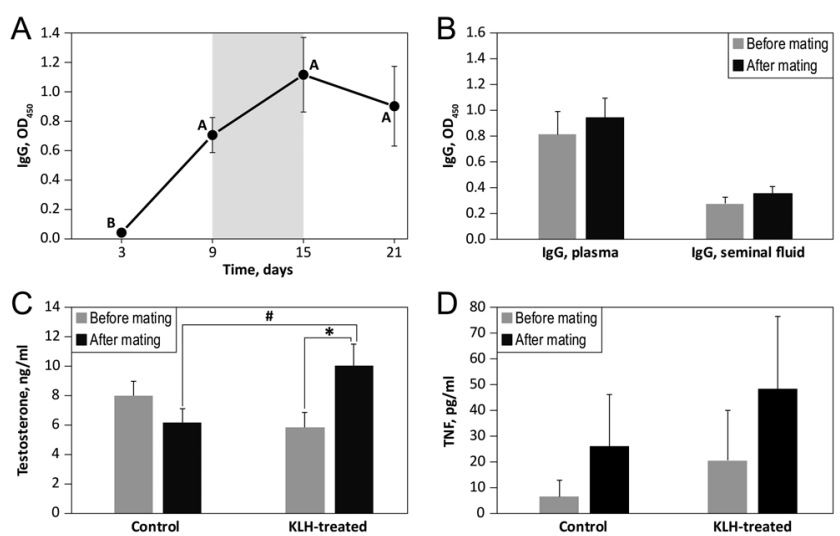

Figure 1 Injection of adult males (fathers) with KLH activates humoral immune response and modulates androgen response to mating with intact females. (A) Dynamics of the anti-KLH IgG in plasma, expressed as $\mathrm{OD}_{450}$. Letters indicate statistically significant differences $(P<0.05)$ according to LSD test. The time of mating after the $\mathrm{KLH}$ treatment is indicated by grey bar. (B) Concentration of the anti-KLH IgG in plasma and seminal fluid before (grey bars) and after (black bars) the mating. The levels of anti-KLH IgG correlated positively in blood and seminal plasma $(r=0.54, P<0.005, n=25)$. (C) Plasma testosterone in control and immunised males before and after the mating. Statistically significant differences according to Mann-Whitney $U$ test: ${ }^{\sharp} P<0.025$ for comparison of immunised and control males after the mating, and $* P=0.037$ for comparison of immunised males before and after the mating. (D) TNF in the seminal fluid of control and immunised males before and after the mating. No statistically significant differences were detected. control males. Specifically, plasma testosterone was significantly increased after mating in the immunised, but not in the control males (Fig. 1C). The anti-KLH IgG level in seminal fluid was positively correlated with plasma testosterone $(r=0.56, P=0.027, n=15)$ after mating. According to a non-parametric twoway ANOVA (Friedman test), the concentration of TNF in the seminal fluid was independent from the immunisation, mating, and the interaction of these two factors (Fig. 1D). Both immunisation and mating did not significantly affect the characteristics of spermatozoa (data not shown).

\section{Mating rate and pre- and postnatal development of the offspring}

Within 6 days (9-15 days after the immunisation) of mating, the control males fertilised 21 females $(70.0 \pm 8.4 \%)$, and the antigen-stimulated males fertilised 25 females $(83.3 \pm 5.0 \%)$. The difference between these groups was not statistically significant. At the same time, the rate of cumulative reproductive output, estimated as the proportion of progeny produced from the start of mating, was significantly different between the control and KLH groups (Fig. 2A). In particular, the proportion of progeny conceived after 2 days of coupling in the control group was $55.4 \pm 4.1 \%$, and in the $\mathrm{KLH}$ group, only $35.8 \pm 3.6 \%$ was conceived after 2 days $(t=3.6$, $d f=322, P<0.001$, Student's $t$-test). However, in the following days (3-6) the proportions were opposite with $44.6 \pm 4.1 \%$ in the control group and $64.2 \pm 3.6 \%$ in the group of immunised males $(t=3.6, d f=322$, $P<0.001$, Student's $t$-test). In terms of absolute numbers, the number of progeny produced in the first 2 days was not significantly different between control and antigen-stimulated males (Fig. 2A). Between days 3 and 6 , the immunised males conceived more progeny than the control males $\left(\chi^{2}=6.04, P=0.014\right)$ (Fig. 2A). In addition, the immunised males who successfully fertilised two females had a higher level of anti-KLH IgG in the blood and seminal plasma than the males who fertilised only one female (Table 1 ). As a result, the total number of progeny conceived by immunised males was significantly correlated with the concentration of anti-KLH IgG in the seminal fluid $(r=0.67, P=0.006$, $n=15$ ), and almost significantly correlated with the concentration of anti-KLH IgG in the blood plasma $(r=0.44, P=0.098, n=15)$. The concentration of TNF in the seminal fluid did not differ between males who mated either one or two females (Table 1).

The average number of live embryos per female (control group: $7.78 \pm 0.36$; KLH group: $7.92 \pm 0.26$ ), as well as traits of embryonic development, including foetal mass, placenta weight, and fetoplacental index, were not significantly different between the groups (Fig. $2 \mathrm{~B})$. Nonetheless, the individual variations in immune responses to the $\mathrm{KLH}$ injection were correlated with 


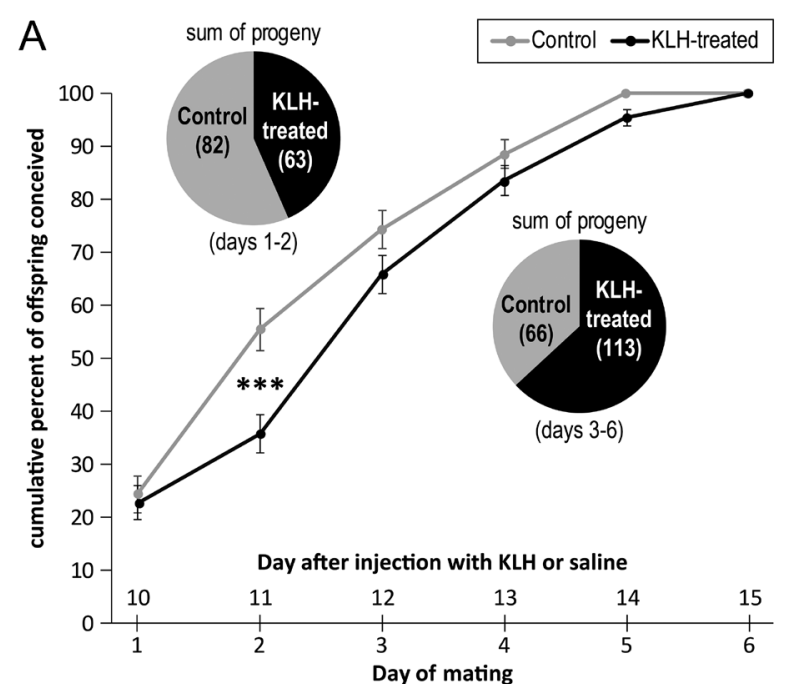

B
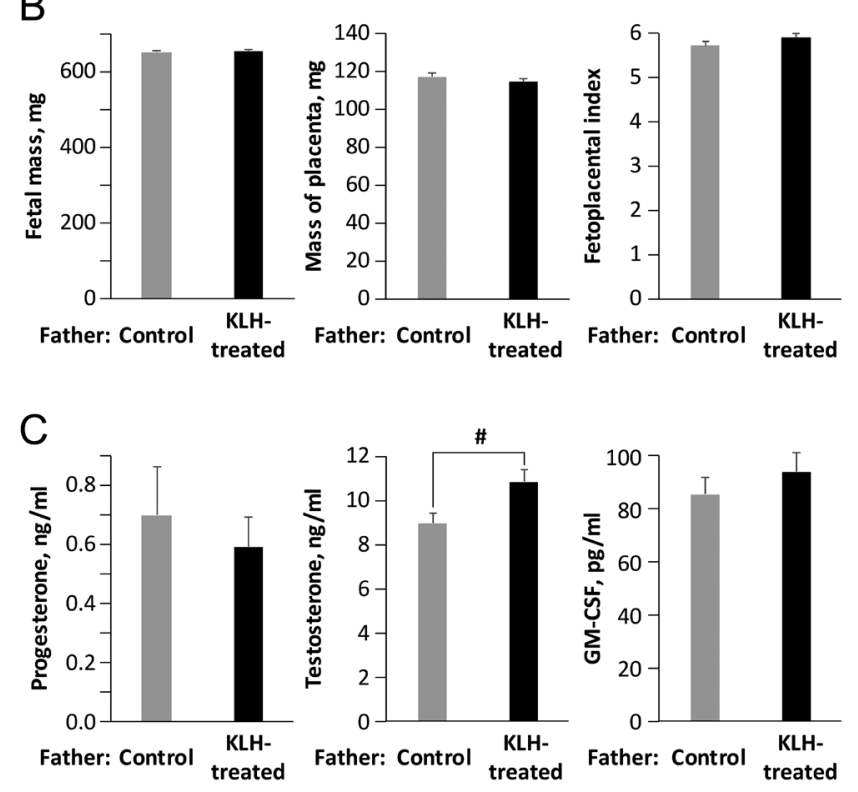

Figure 2 Immunisation of fathers delays the fertile mating and results in higher concentration of testosterone in amniotic fluid. (A) Cumulative curve for proportion of progeny conceived by the control (grey line) or the KLH-treated (black line) males at different times from the moment of mating with two mature females. Time after injection with the KLH or saline is indicated on $\mathrm{x}$-axis above. Student's $t$-test $(d f=322),{ }^{* * *} P<0.001$ for comparison of the control and immunised males. Insets: circle diagrams showing the number of live embryos (sum of progeny) conceived by the control (grey segments) or KLH-treated (black segments) males at the first (1-2 days) and the second (3-6 days) stage of the mating. The number of embryos produced by the control males did not differ from that of the immunised males $\left(\chi^{2}=1.38, P=0.24\right)$ at days $1-2$. In the following days (3-6), immunised males conceived more progeny than control males $\left(\chi^{2}=6.04, P=0.014\right)$. (B) Fetal mass, mass of placenta and fetoplacental ratio at day 16 of the pregnancy for the control (grey bars) and KLH-treated (black bars) fathers. (C) Concentrations of hormones (progesterone and testosterone) and GM-CSF cytokine in the amniotic fluid at day 16 of the pregnancy in females mated by the control or KLH-treated males. Mann-Whitney $U$ test, ${ }^{\sharp} P=0.035$, indicates significant differences in amniotic testosterone levels. the placenta weight $(r=0.28, P=0.004$ for anti-KLH IgG in plasma, and $r=0.22, P=0.02$ for anti-KLH IgG in seminal fluid, $n=108$ ) and fetoplacental index $(r=-0.21, P=0.03$ for anti-KLH IgG in plasma, $n=108)$. The concentrations of progesterone and testosterone in the blood plasma were similar in each group (data not shown). Progesterone and GM-CSF in the amniotic fluid (Fig. 2C) were also similar between groups. At the same time, the level of amniotic testosterone was higher in the females mated by the antigen-stimulated males compared with the females mated by the control males (Fig. 2C).

The average number of newborns was nearly equal in the females mated by control $(7.80 \pm 0.55)$ and antigen-treated $(7.44 \pm 0.34)$ males (Fig. 3A). Interestingly, however, the percentage of newborns that died before weaning was significantly higher in the females of the control group $(39.74 \pm 5.54 \%)$ compared with that in the females of the KLH group $(17.90 \pm 4.68 \%)(t=2.31, d f=143, P<0.05$, Student's $t$-test). These differences were due to a combination of two effects, including (a) the withdrawal of feeding was detected in three females from the control and one female from the KLH group, and (b) the death of newborns, which was $16.1 \pm 4.9 \%$ in the control group and $8.1 \pm 3.6 \%$ in the KLH group (Fig. 3B). These pre-weaning mortalities and whole litter losses were comparable with other studies of $\mathrm{C} 57 \mathrm{BL} / 6 \mathrm{~J}$ mice (Cooper et al. 2007, Whitaker et al. 2009, Weber et al. 2013). The average number of pups fed by the females mated by the control males $(6.71 \pm 0.86)$ did not differ from that mated by the antigen-stimulated males $(6.88 \pm 0.35)$.

The body mass of the offspring at weaning (3 weeks of age) was higher in the control group compared with the KLH group (Fig. 3C). Since the litter size just before weaning was negatively correlated with the body mass of 3-week-old progeny $(r=-0.59, P<0.001$, $n=102$ ), the ANCOVA was used to assess the statistical significance between-group differences. ANCOVA with the litter size as the covariate revealed a reliable influence of the father's immune status $($ control/KLH) on the body mass of the progeny $\left(F_{1,97}=38.88, P<0.001\right)$. The effects of sex and sex $x$ group interaction were statistically insignificant. Therefore, the data obtained from the offspring of both sexes were combined (Fig. 3C). Of note, in those litters in which partial losses of progeny were recorded, the body weight of the fed pups was significantly higher $(9.58 \pm 0.23, n=27)$ than in the litters with no losses during the suckling period $(8.12 \pm 0.12, n=75)$. The statistical significance of these differences was confirmed by a three-way ANOVA, in which the $F$ criterion had the following values: for the factor of feeding (complete feeding vs incomplete feeding) $-F_{1,94}=30.18, P<0.001$; experimental group (control/KLH) $-F_{1,94}=24.92, P<0.001$; and the sex of the progeny - insignificant. 
Table 1 Differences in immune response to the injection of KLH for males, which successfully fertilized either one or two females.

\begin{tabular}{|c|c|c|c|}
\hline \multirow[b]{2}{*}{ Traits } & \multicolumn{2}{|c|}{ Number of fertilized females, mean \pm S.E. } & \multirow[b]{2}{*}{ Mann-Whitney test } \\
\hline & One $(n=6)$ & Two $(n=9)$ & \\
\hline Anti-KLH IgG in seminal fluid, $\mathrm{OD}_{405}$ & $0.52 \pm 0.17$ & $1.23 \pm 0.18$ & $P=0.021^{*}$ \\
\hline Anti-KLH IgG in plasma, $\mathrm{OD}_{405}$ & $0.22 \pm 0.03$ & $0.45 \pm 0.07$ & $P=0.011^{*}$ \\
\hline TNF in seminal fluid, $\mathrm{pg} / \mathrm{mL}$ & $18.96 \pm 9.49$ & $68.16 \pm 46.36$ & NS \\
\hline
\end{tabular}

${ }^{*} P<0.05 ; \mathrm{NS}$, non-significant.

\section{MRS of neurometabolites in the amygdala and cortex of male offspring}

The MRS of the amygdala in adult male offspring (Fig. 4A) revealed that the sons of immunised fathers had lower levels of gamma-aminobutyric acid (GABA), alanine, creatine, and phosphocreatine $(\mathrm{Cr}+\mathrm{Pcr})$ compared with the male progeny of control fathers (Fig. 4B). In contrast, the amounts of glutamate and glutamine (GLX) were significantly higher in the offspring of the KLH group than in the control group (Fig. 4B). As a result, there was a significant shift in the ratio of inhibitory over excitatory neurotransmitters (GABA/GLX), which was $12.81 \pm 2.78$ in the offspring of control fathers and $0.34 \pm 0.19$ in the offspring of antigen-stimulated fathers $(t=3.95, d f=8$, $P=0.005$, Student's $t$-test). In contrast to the amygdala, the profile of neurometabolites in the cerebral cortex was the same among the progeny of these two groups (Fig. 4C).

\section{Immunity of the male offspring}

The body weight and the mass indexes of the main immunocompetent organs (thymus and spleen) were measured in both groups of the male adult offspring, who were either treated with KLH or untreated. From the results of a two-way ANOVA (Supplementary
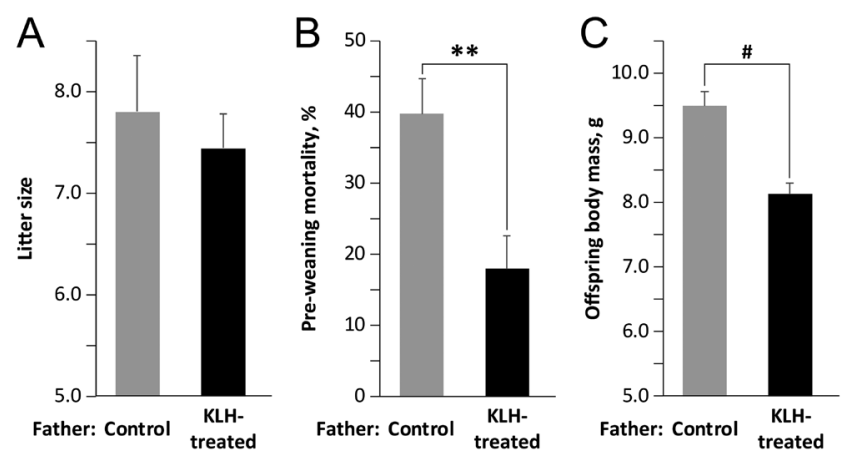

Figure 3 Mating by immunised males does not affect litter size, but reduces postnatal mortality and body mass of newborns. (A) Average number of newborns per female. (B) Average percentage of pups died from the delivery to the weaning. Student's t-test $(d f=143), * * P<0.01$ for comparison of the groups of females mated by the immunised and control males. (C) Average body mass of the pups at weaned time. Statistically significant differences between the groups (offspring of the control and immunised fathers), ${ }^{*} P<0.001$ according to the ANCOVA with the group and gender as the factors and the number of embryos as the covariate $\left(F_{1,97}=38.88, P<0.001\right)$.
Table 1, see section on supplementary materials given at the end of this article), neither immunisation of the fathers (group-of-progeny) nor immunisation of the sons had statistically significant effects on body weight, which was $23.4 \pm 0.5 \mathrm{~g}$ in the control group $(n=14)$ and $23.1 \pm 0.5 \mathrm{~g}$ in the $\mathrm{KLH}$ group $(n=14)$. At the same time, the offspring of antigen-stimulated fathers had significantly higher mass indexes of the thymus and spleen than the offspring of the control group (Supplementary Tables 1 and 2). Since the effects of the immunisation of offspring and the interaction of factors (group-of-progeny $\times$ immunisation) were insignificant, the data on mass indexes of immunocompetent organs in offspring who were treated with $\mathrm{KLH}$ or untreated were combined (Fig. 5A). Despite the large size of the thymus and spleen, the progeny of the control and $\mathrm{KLH}$ treated fathers showed the same values of the specific immunoglobulin (IgG), which were determined in blood plasma samples 9 days after the KLH injection (Fig. 5B).

\section{Androgens, gonad system, and spermatozoa of the male offspring}

A two-way ANOVA of mass indexes showed a significant effect of the male offspring group on the size of preputial glands (Supplementary Table 3), which were larger in the progeny of antigen-stimulated fathers (Fig. 6A and Supplementary Table 4). This result was consistent with the observed intergroup difference in plasma testosterone, which was significantly higher in the progeny of antigen-stimulated fathers $(18.9 \pm 3.4 \mathrm{ng} / \mathrm{mL})$ compared with the progeny of control fathers $(7.7 \pm 2.6$ $\mathrm{ng} / \mathrm{mL})(Z=2.78, P=0.016$, Mann-Whitney $U$ test). The interaction of factors (group-of-progeny $\times$ immunisation) also significantly influenced testosterone variations (Fig. $6 \mathrm{~B}$ ). Immunisation (injection with $\mathrm{KLH}$ ) of the offspring of control fathers caused a significant decrease in testosterone concentration $(Z=2.04, p=0.04$, MannWhitney $U$ test), while immunisation of the progeny of antigen-stimulated males tended to increase it, although this result was insignificant.

All characteristics of spermatozoa were comparable with the literature (Fiedler et al. 2013). Despite statistically significant differences in the plasma testosterone of the male offspring of control and immunised fathers before and after the KLH administration (Fig. 6B), these groups had rather similar characteristics of sperm morphology, count, and motility (Fig. 7 and Supplementary Table 

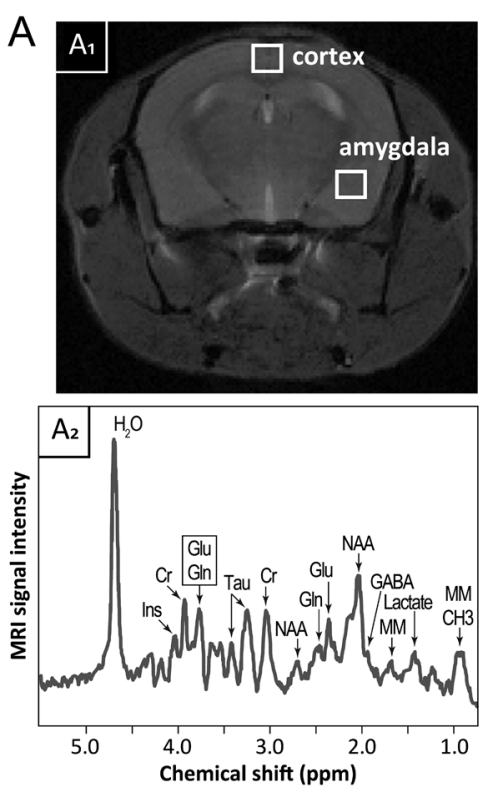
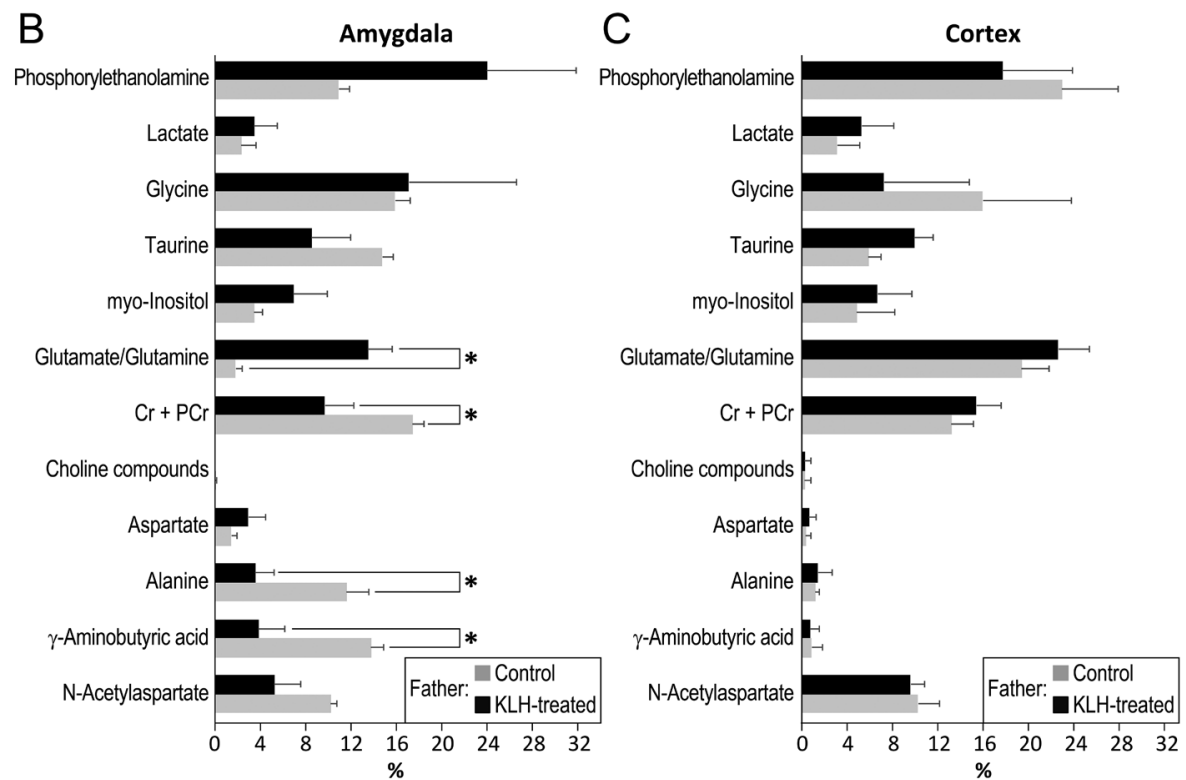

Figure 4 Prevalence of excitatory (glutamate/glutamine - GLX) over inhibitory (GABA) neurotransmitters in the amygdala of the adult offspring of immunised fathers. (A) The positions of the voxels in cortex and amygdala on the axial section of mouse brain $\left(\mathrm{A}_{1}\right)$ and the typical in vivo MRS spectrum from the mouse amygdala $\left(\mathrm{A}_{2}\right)$. Ins, myo-inositol; Cr, creatine; Glu, glutamate; Gln, glutamine; Tau, taurine; NAA, N-acetylaspartate; GABA, $\gamma$-aminobutyric acid; MM, macromolecules. (B, C). The relative levels of neurometabolites (\% of the total) in the amygdala (B) and in the cerebral cortex $(C)$ of the adult offspring of control (grey bars) and immunised (black bars) fathers. Student's $t$-test $(d f=8),{ }^{*} P<0.05$ for comparison of the offspring conceived by immunised and control males.
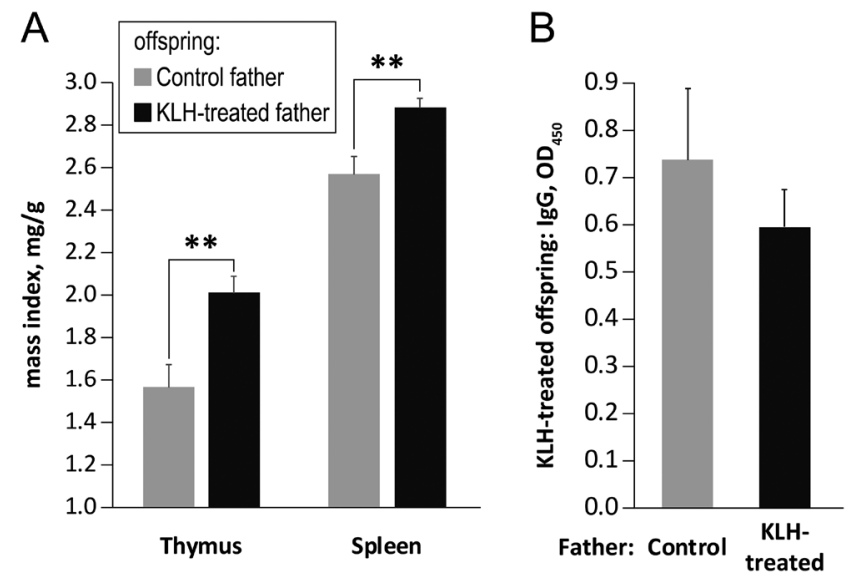

Figure 5 Mating by immunised males increases mass indexes of the thymus and spleen in adult offspring, but does not affect their antibody response to a foreign antigen. (A) Mass indexes $(\mathrm{mg} / \mathrm{g})$ of the thymus and spleen in the offspring of immunised and control fathers. Two-way ANOVA revealed significant effect of the progeny group $\left(F_{1,24}=11.038, P=0.003\right)$. Since, there were no significant effects of injection of the male offspring with the $\mathrm{KLH}$ and interaction of these two factors (Supplementary Table 1), the data of mass indexes of the $\mathrm{KLH}$ treated and non-treated offspring were combined. Student's $t$-test $(d f=26),{ }^{*} * P<0.01$ for the differences between the offspring of immunised and control fathers. (B) Plasma concentration, expressed as $\mathrm{OD}_{450}$, of the anti-KLH in the offspring of immunised and control fathers challenged with the foreign antigen (the KLH).
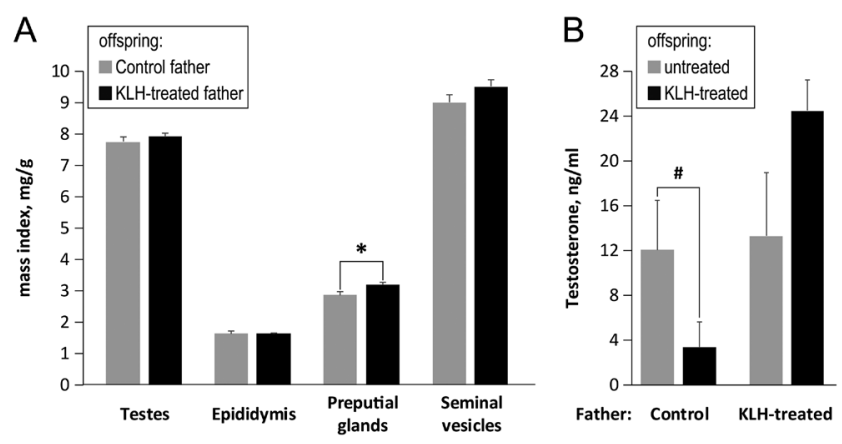

Figure 6 Offspring of immunised fathers maintain testosterone levels after the injection with $\mathrm{KLH}$ and have higher mass index of preputial glands in comparison with the control. (A) Mass indexes ( $\mathrm{mg} / \mathrm{g}$ ) of androgen dependent organs in the offspring of control and immunised fathers. Since there were no significant effects of the injection with $\mathrm{KLH}$ and interaction of the progeny group (Supplementary Table 3), the data of mass indexes were combined for $\mathrm{KLH}$ treated and untreated offspring. Significant effect of the progeny group (control and immunised fathers) was confirmed by the two-way ANOVA $\left(F_{1,24}=4.335, P=0.048\right)$. Student's $t$-test $(d f=26)$, $* P<0.05$ for the differences between the offspring of immunised and control fathers. (B) Plasma testosterone in the offspring of control and immunised fathers injected with the saline (Control) or challenged with the KLH (Immunisation). Non-parametric two-way ANOVA (Friedman test) revealed significant effects of the progeny group $\left(F_{1,24}=7.725, P=0.010\right)$, and interaction of the progeny group and immunisation of offspring $\left(F_{1,24}=6.116, P=0.021\right)$. Effect of the offspring immunisation was insignificant $\left(F_{1,24}=0.097, P=0.757\right)$. Mann-Whitney $U$ test, ${ }^{\sharp} P=0.04$ for comparison of the testosterone levels before and after the immunisation in the offspring of control fathers. 
5). However, bidirectional testosterone responses to the antigenic stimulus in the offspring of immunised and control fathers (Fig. 6B) subsequently affected the spermatozoa VAP and VSL. These were slightly decreased in the progeny of control fathers and modestly increased in the progeny of immunised fathers after the $\mathrm{KLH}$ administration (Fig. 7C). A two-way ANOVA showed statistical significance for the effect of factor interaction (group-of-progeny and immunisation) on VAP and VSL (Supplementary Table 5). The analysis of correlations between plasma testosterone and sperm characteristics confirmed the dependence of spermatozoa concentration and velocity indexes on the level of androgens in the blood (Table 2).

\section{Discussion}

Although the main limitation of this study is the use of a model antigen $(\mathrm{KLH})$ to induce an immune response, these results suggest that the immunisation of male mice before mating modulates their reproductive characteristics. In particular, we noted distinct dynamics in the rate of cumulative reproductive output for immunised fathers. During the first days of mating, the immunised males produced less progeny compared with the control fathers, but in the following days, they outperformed. An initially low reproductive output might be associated with reduced behavioural responses of the immunised male mice to intact females (Moshkin et al. 2010). In the later stages of mating, an increase in the reproductive output of immunised males was consistent with increasing levels of plasma testosterone. For example, the androgen response to the immunisation was positively correlated with the humoral immune response, and in particular, with the concentration of anti-KLH IgG in the seminal fluid. Immunoglobulins modulate cytokine composition of the seminal fluid (Pillay et al. 2018), and thus, can exert multiple effects on the female reproductive tract mediating embryo implantation, growth, and immune response against foetal (paternal) antigens (Chaouat et al. 2007, Rametse et al. 2018, Robertson et al. 2018, Maslennikova et al. 2019).

Mating with immunised males led to an increase in testosterone levels in the amniotic fluid, a reduction in newborn mortality during nursing, and a decrease in the body mass of weaned pups. It should be noted that in the litters with partial losses of progeny, the body mass of the remaining pups was significantly higher than in the litters without losses. Thus, newborn deaths were not associated with a lactation deficit. Together, this indicates that the transmission of paternal antigenic stimuli, evidenced here by the anti-KLH IgG level in the seminal fluid, can have a positive impact on pregnancy and nursing. Other changes in the cellular and humoral composition of the seminal fluid of immunised fathers might also be responsible for the observed effects (Schjenken \& Robertson 2015).

In addition to reproductive output, a one-time immunisation of fathers modulated the phenotype of their adult offspring. This manifested as changes in the amygdala neurometabolic profile, the size of immune organs, plasma testosterone, and spermatogenesis. A paternal effect on brain metabolism was detected in the amygdala, but not in the cortex. Some of the phenotypic changes in the progeny of immunised fathers might be interrelated. For example, the predominance of excitatory (glutamate/glutamine) neurotransmitters over inhibitory (GABA, alanine) ones in the amygdala may be a factor that contributes to the higher level of androgens in the offspring of immunised fathers. Indeed, mice with a knockout of the metabotropic glutamate receptor subtype 7 gene are characterised by a lower blood testosterone level compared with wild-type mice (Masugi-Tokita et al. 2018). Likewise, prenatal testosterone affects the development of the amygdala, which has been shown in both experimental and clinical studies (Kerchner et al. 1995, Lombardo et al. 2012). The action of androgens on brain structures might be sensed through a family of androgen and oestrogen receptors, which are expressed in the amygdala of embryos and mature individuals (Sarkey et al. 2008, Filová et al. 2013,
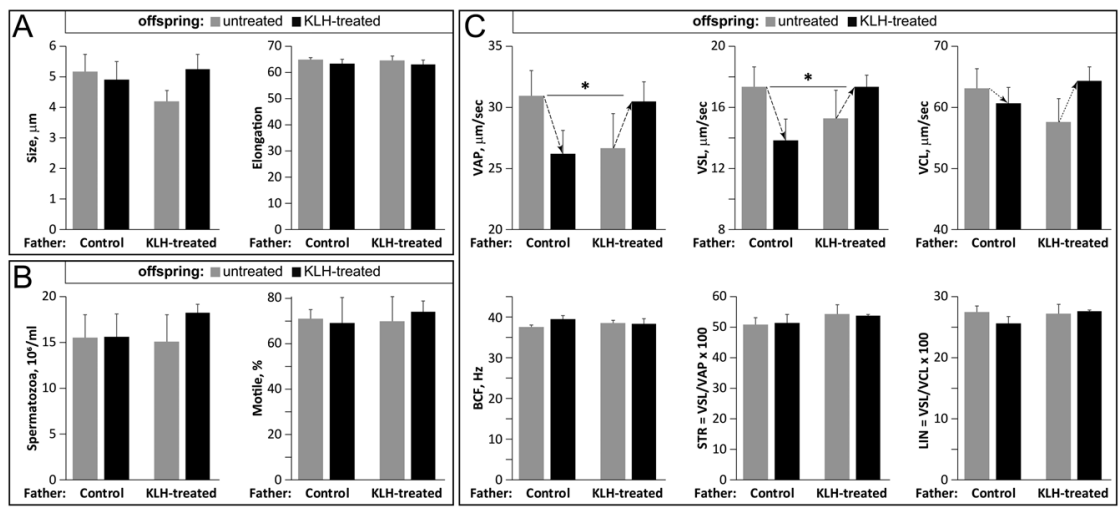

Reproduction (2020) 160 117-127
Figure 7 Mating by immunised males does not affect sperm morphology and concentration in offspring, but modulates a response of sperm velocity to the immunisation. (A) Average size and shape (elongation) of spermatozoa head in the offspring of control and immunised fathers before (grey bars) and after (black bars) their immunisation. (B) Concentration and \% of motile spermatozoa in caudal epididymis. (C) Velocities and trajectories of movement of spermatozoa. Effects of the progeny group and immunisation of offspring were analyzed by two-way ANOVA (Supplementary Table 5). *Statistically significant effects of interactions of these two factors on VAP $\left(F_{1,24}=4.085\right.$, $P=0.050)$ and $\operatorname{VSL}\left(F_{1,24}=4.075, P=0.050\right)$. 
Table 2 Correlations of sperm characteristics with the plasma testosterone in male offspring.

\begin{tabular}{lcc}
\hline Traits & $\boldsymbol{r}$ & $\boldsymbol{P}$ \\
\hline Size & 0.262 & $\mathrm{NS}$ \\
Elongation & 0.028 & $\mathrm{NS}$ \\
Spermatozoid concentration & 0.423 & $0.025^{*}$ \\
Percent of motile & 0.102 & $\mathrm{NS}$ \\
VAP & 0.386 & $0.042^{*}$ \\
VSL & 0,503 & $0.006^{* *}$ \\
VCL & 0.294 & $\mathrm{NS}$ \\
BSF & -0.037 & $\mathrm{NS}$ \\
STR & 0.279 & $\mathrm{NS}$ \\
LIN & 0.402 & $0.034^{*}$ \\
\hline
\end{tabular}

${ }^{*} P<0.05,{ }^{* *} P<0.01, \mathrm{NS}$, non-significant.

Cisternas et al. 2015). In addition, the predominance of excitatory neurotransmitters over inhibitory ones in the amygdala might modulate the social behaviour of the male offspring of immunised fathers, including sexual activity and the ability to dominate in social competition with other males (Watanabe \& Yamamoto 2015, Dulka et al. 2018).

The paternal effect on gonadal function was most evident under the antigenic challenge of the progeny. The KLH injection caused a decrease in plasma testosterone in the offspring of control fathers. However, in the offspring of antigen-stimulated fathers, there was an opposite trend. The differences in androgen response to the antigenic challenge in the studied offspring groups were paralleled with the changes in sperm velocity parameters, which was in agreement with other observations (Guvvala et al. 2016, Dardmeh et al. 2017). Thus, the ability of the adult progeny of antigenstimulated fathers to maintain high levels of androgens after the immunisation preserves spermatozoa velocity parameters under the antigenic challenge. Finally, higher levels of plasma testosterone and excitatory neurotransmitters in the amygdala might promote increased sexual activity in the progeny of immunised fathers (Tzanoulinou et al. 2014, Adams \& Rosenkranz 2016). These suggest that the offspring of immunised fathers might trade-off some of their somatic maintenance to reproductive investment if antigenic pressure prevails in the environment.

Changes in the offspring phenotype of immunised fathers can be attributed to either epigenetic modification of male gametes, the seminal fluid transmission of the paternal signals, or both. Indeed, the time when immunised fathers were mated in this study (9-15 days after the immunisation) coincides with the time of sperm maturation (Khil et al. 2004). Sperm maturation involves massive histone-to-protamine reassembly of the paternal genome, and this process might be affected by the father's immune response (Hazzouri et al. 2000, Morinière et al. 2009, Shirakata et al. 2014). Likewise, the cellular and humoral composition of the seminal fluid, which directly modulates the female reproductive tract, is likely to react to immunisation (Collodel et al. 2015, Robertson \& Sharkey 2016). The significance of the seminal fluid in the determination of the pre- and postnatal phenotype has been convincingly shown in experiments involving the removal of seminal vesicles. This leads to a decrease in fertility, placental hypertrophy, and metabolic disorders, mainly in the male offspring (Bromfield et al. 2014). Furthermore, the paternal experience of defeating in social conflicts is passed to offspring via natural, but not in vitro fertilisation (Dietz et al. 2011). The humoral composition of seminal fluid significantly changes under exposure to various stressors (Meuleman et al. 2015, Dobrakowski et al. 2017, Haimovici et al. 2018). Here, we showed a significant correlation between placental mass and the concentration of anti-KLH IgG in the blood and seminal plasma. Together, these substantiate a pivotal role of seminal fluid in the transgenerational transmission of paternal experiences, although epigenetic signals cannot be excluded.

The paternal effects on the mother's side can be realised through the changes in immune and endocrine support of her pregnancy. We found that the levels of amniotic testosterone were higher in pregnant females mated by the immunised males. The effect of amniotic testosterone on the offspring phenotype has been demonstrated by numerous studies (Sarkar et al. 2008). In particular, males prenatally exposed to androgens had increased circulating testosterone concentrations during adult life (More et al. 2016). This is in agreement with the observed differences in plasma testosterone in the offspring of control and antigen-stimulated fathers in this study. Variations in testosterone in prenatal development might also affect brain structures, including the amygdala (Kerchner et al. 1995, Lombardo et al. 2012), which is consistent with our results.

The adaptive significance of transgenerational transmission of paternal experiences has been widely discussed (Dietz et al. 2011, Rodgers et al. 2013, 2015, Dias \& Ressler 2014). In the framework of the lifehistory theory, this implies the adaptation of offspring to prevailing conditions experienced by their fathers and mothers through the reallocation of available resources. Thus, following this logic, it makes sense to investigate the immune-reproductive trade-off in the offspring of immunised fathers as part of their adaptation strategy. Even though the progeny of antigen-stimulated fathers had higher mass indexes of the thymus and spleen, their humoral response to $\mathrm{KLH}$ did not differ significantly from the control. However, contrary to the control, these offspring maintained high androgen levels after the antigenic challenge with KLH. In other words, the offspring of immunised fathers are capable of equally supporting both immune and reproductive capacities under the antigenic pressure, while the offspring of inexperienced fathers reduce their reproduction effort. 


\section{Supplementary materials}

This is linked to the online version of the paper at https://doi. org/10.1530/REP-19-0360.

\section{Declaration of interest}

The authors declare that there is no conflict of interest that could be perceived as prejudicing the impartiality of the research reported.

\section{Funding}

The studies are supported by the budget project (No. AAAA-A17-17072710029-7; 0259-2019-0004) and implemented using the equipment of the Center for Genetic Resources of Laboratory Animals at ICG SB RAS, supported by the Ministry of Education and Science of Russia (Unique identifier of the project RFMEFI62119X0023); the Russian Foundation for Basic Research (18-34-00493 mol.a).

\section{Author contribution statement}

L G conceived and designed study and wrote manuscript. M A performed mating schemes and assessed reproductive output. G K analyzed endocrine status of pregnant females. S M analyzed immunity and androgen functions of progeny. A R performed MRS of amygdala and cortex. Y G participated in data collection and assisted in interpretation of data. $Y M$ revised manuscript and assisted in interpretation of data. M M statistically treated experimental data and provided critical discussion.

\section{References}

Adams T \& Rosenkranz JA 2016 Social isolation during postweaning development causes hypoactivity of neurons in the medial nucleus of the male rat amygdala. Neuropsychopharmacology 41 1929-1940. (https:// doi.org/10.1038/npp.2015.364)

Arakawa H, Arakawa K \& Deak T 2010 Sickness-related odor communication signals as determinants of social behavior in rat: a role for inflammatory processes. Hormones and Behavior 57 330-341. (https://doi.org/10.1016/j.yhbeh.2010.01.002)

Bromfield JJ, Schjenken JE, Chin PY, Care AS, Jasper MJ \& Robertson SA 2014 Maternal tract factors contribute to paternal seminal fluid impact on metabolic phenotype in offspring. PNAS 111 2200-2205. (https://doi. org/10.1073/pnas.1305609111)

Byers SL, Wiles MV, Dunn SL \& Taft RA 2012 Mouse estrous cycle identification tool and images. PLOS ONE 7 e35538. (https://doi. org/10.1371/journal.pone.0035538)

Chaouat G, Dubanchet S \& Ledée N 2007 Cytokines: important for implantation? Journal of Assisted Reproduction and Genetics 24 491-505. (https://doi.org/10.1007/s10815-007-9142-9)

Cisternas CD, Tome K, Caeiro XE, Dadam FM, Garcia-Segura LM \& Cambiasso MJ 2015 Sex chromosome complement determines sex differences in aromatase expression and regulation in the stria terminalis and anterior amygdala of the developing mouse brain. Molecular and Cellular Endocrinology 414 99-110. (https://doi.org/10.1016/j.mce.2015.07.027)

Collodel G, Moretti E, Brecchia G, Kuželová L, Arruda J, Mourvaki E \& Castellini C 2015 Cytokines release and oxidative status in semen samples from rabbits treated with bacterial lipopolysaccharide. Theriogenology 83 1233-1240. (https://doi.org/10.1016/j.theriogenology.2015.01.008)
Cooper JC, Dealtry GB, Ahmed MA, Arck PC, Klapp BF, Blois SM \& Fernández N 2007 An impaired breeding phenotype in mice with a genetic deletion of beta- 2 microglobulin and diminished MHC Class I expression: role in reproductive fitness. Biology of Reproduction 77 274-279. (https://doi.org/10.1095/biolreprod.106.057125)

Dardmeh F, Alipour H, Gazerani P, Van Der Horst G, Brandsborg E \& Nielsen HI 2017 Lactobacillus rhamnosus PB01 (DSM 14870) supplementation affects markers of sperm kinematic parameters in a diet-induced obesity mice model. PLOS ONE 12 e0185964. (https://doi. org/10.1371/journal.pone.0185964)

Dias BG \& Ressler KJ 2014 Parental olfactory experience influences behavior and neural structure in subsequent generations. Nature Neuroscience 17 89-96. (https://doi.org/10.1038/nn.3594)

Dietz DM, Laplant Q, Watts EL, Hodes GE, Russo SJ, Feng J, Oosting RS, Vialou V \& Nestler EJ 2011 Paternal transmission of stress-induced pathologies. Biological Psychiatry 70 408-414. (https://doi.org/10.1016/j. biopsych.2011.05.005)

Dobrakowski M, Kasperczyk S, Horak S, Chyra-Jach D, Birkner E \& Kasperczyk A 2017 Oxidative stress and motility impairment in the semen of fertile males. Andrologia 49 e12783. (https://doi.org/10.1111/ and.12783)

Dominguez J, Riolo JV, Xu Z \& Hull EM 2001 Regulation by the medial amygdala of copulation and medial preoptic dopamine release. Journal of Neuroscience 21 349-355. (https://doi.org/10.1523/ jneurosci.21-01-00349.2001)

Dulka BN, Bress KS, Grizzell JA \& Cooper MA 2018 Social dominance modulates stress-induced neural activity in medial prefrontal cortex projections to the basolateral amygdala. Neuroscience 388 274-283. (https://doi.org/10.1016/j.neuroscience.2018.07.042)

Faivre B, Grégoire A, Préault M, Cézilly F \& Sorci G 2003 Immune activation rapidly mirrored in a secondary sexual trait. Science $\mathbf{3 0 0} 103$. (https://doi.org/10.1126/science.1081802)

Fiedler SE, Dudiki T, Vijayaraghavan S \& Carr DW 2013 Loss of R2D2 proteins ROPN1 and ROPN1L causes defects in murine sperm motility, phosphorylation, and fibrous sheath Integrity1. Biology of Reproduction 88 41. (https://doi.org/10.1095/biolreprod.112.105262)

Filová B, Ostatníková D, Celec P \& Hodosy J 2013 The effect of testosterone on the formation of brain structures. Cells, Tissues, Organs 197 169-177. (https://doi.org/10.1159/000345567)

Gerlinskaya LA, Maslennikova SO, Anisimova MV, Feofanova NA, Zavjalov EL, Kontsevaya GV, Moshkin YM \& Moshkin MP 2017 Modulation of embryonic development due to mating with immunized males. Reproduction, Fertility, and Development 29 565-574. (https:// doi.org/10.1071/RD15173)

Gerlinskaya LA, Shnayder EP, Dotsenko AS, Maslennikova SO, Zavjalov EL \& Moshkin MP 2012 Antigen-induced changes in odor attractiveness and reproductive output in male mice. Brain, Behavior, and Immunity 26 451-458. (https://doi.org/10.1016/j.bbi.2011.11.010)

Gresham R, Li S, Adekunbi DA, Hu M, Li XF \& TO'Byrne KT 2016 Kisspeptin in the medial amygdala and sexual behavior in male rats. Neuroscience Letters 627 13-17. (https://doi.org/10.1016/j.neulet.2016.05.042)

Guvvala PR, Sellappan S \& Parameswaraiah RJ 2016 Impact of arsenic(V) on testicular oxidative stress and sperm functional attributes in Swiss albino mice. Environmental Science and Pollution Research International 23 18200-18210. (https://doi.org/10.1007/s11356-016-6870-3)

Haimovici F, Anderson JL, Bates GW, Racowsky C, Ginsburg ES, Simovici D \& Fichorova RN 2018 Stress, anxiety, and depression of both partners in infertile couples are associated with cytokine levels and adverse IVF outcome. American Journal of Reproductive Immunology 79 e12832. (https://doi.org/10.1111/aji.12832)

Hazzouri M, Pivot-Pajot C, Faure AK, Usson Y, Pelletie R, Sèle B, Khochbin S \& Rousseaux S 2000 Regulated hyperacetylation of core histones during mouse spermatogenesis: involvement of histonedeacetylases. European Journal of Cell Biology 79 950-960. (https://doi. org/10.1078/0171-9335-00123)

Kerchner M, Malsbury CW, Ward OB \& Ward IL 1995 Sexually dimorphic areas in the rat medial amygdala: resistance to the demasculinizing effect of prenatal stress. Brain Research 672 251-260. (https://doi. org/10.1016/0006-8993(94)01378-U)

Khil PP, Smirnova NA, Romanienko PJ \& Camerini-Otero RD 2004 The mouse $X$ chromosome is enriched for sex-biased genes not subject to 
selection by meiotic sex chromosome inactivation. Nature Genetics 36 642-646. (https://doi.org/10.1038/ng1368)

Lombardo MV, Ashwin E, Auyeung B, Chakrabarti B, Taylor K, Hackett G, Bullmore ET \& Baron-Cohen S 2012 Fetal testosterone influences sexually dimorphic gray matter in the human brain. Journal of Neuroscience 32 674-680. (https://doi.org/10.1523/JNEUROSCI.4389-11.2012)

Luu H \& Tate AT 2017 Recovery and immune priming modulate the evolutionary trajectory of infection-induced reproductive strategies. Journal of Evolutionary Biology 30 1748-1762. (https://doi.org/10.1111/jeb.13138)

Maslennikova SO, Gerlinskaya LA, Kontsevay GV, Anisimova MV, Nedospasov SA, Feofanova NA, Moshkin MP \& Moshkin YM 2019 TNF $\alpha$ is responsible for the canonical offspring number-size trade-off. Scientific Reports 9 4568. (https://doi.org/10.1038/s41598-019-38844-9)

Masugi-Tokita M, Yoshida T, Kageyama S, Kawata M \& Kawauchi A 2018 Metabotropic glutamate receptor subtype 7 has critical roles in regulation of the endocrine system and social behaviours. Journal of Neuroendocrinology 30 e12575. (https://doi.org/10.1111/jne.12575)

McNamara JM \& Houston Al 1996 State-dependent life histories. Nature 380 215-221. (https://doi.org/10.1038/380215a0)

Meuleman T, Snaterse G, van Beelen E, Anholts JDH, Pilgram GSK, van der Westerlaken LAJ, Eikmans M \& Claas FHJ 2015 The immunomodulating effect of seminal plasma on T cells. Journal of Reproductive Immunology 110 109-116. (https://doi.org/10.1016/j.jri.2015.01.012)

More AS, Mishra JS, Gopalakrishnan K, Blesson CS, Hankins GD \& Sathishkumar K 2016 Prenatal testosterone exposure leads to gonadal hormone-dependent hyperinsulinemia and gonadal hormoneindependent glucose intolerance in adult male rat offspring. Biology of Reproduction 94 5. (https://doi.org/10.1095/biolreprod.115.133157)

Morinière J, Rousseaux S, Steuerwald U, Soler-López M, Curtet S, Vitte AL, Govin J, Gaucher J, Sadoul K, Hart DJ et al. 2009 Cooperative binding of two acetylation marks on a histone tail by a single bromodomain. Nature 461 664-668. (https://doi.org/10.1038/nature08397)

Moshkin M, Gerlinskaya L, Morozova O, Bakhvalova V \& Evsikov V 2002 Behaviour, chemosignals and endocrine functions in male mice infected with tick-borne encephalitis virus. Psychoneuroendocrinology 27 603-608. (https://doi.org/10.1016/S0306-4530(01)00096-8)

Moshkin MP, Kondratyuk EY, Litvinova EA \& Gerlinskaya LA 2010 The activation of specific immunity in male mice stimulates fertility of their breeding partners: the phenomenon of Lot's daughters. Zhurnal Obshchei Biologii 71 425-435. (https://doi.org/10.1134/s2079086411010063)

Moshkin MP, Akulov AE, Petrovski DV, Saik OV, Petrovskiy ED, Savelov AA \& Koptyug IV 2014 Proton magnetic resonance spectroscopy of brain metabolic shifts induced by acute administration of 2-deoxy-d-glucose and lipopolysaccharides. NMR in Biomedicine 27 399-405. (https://doi. org/10.1002/nbm.3074)

Nelson JF, Felicio LS, Randall PK, Sims C \& Finch CE 1982 A longitudinal study of estrous cyclicity in aging $\mathrm{C} 57 \mathrm{BL} / 6 \mathrm{~J}$ mice: I. Cycle frequency, length and vaginal cytology. Biology of Reproduction 27 327-339. (https://doi.org/10.1095/biolreprod27.2.327)

Pillay T, Sobia P, Olivier AJ, Narain K, Liebenberg LJP, Ngcapu S, Mhlongo M, Passmore JS, Baxter C \& Archary D 2018 Semen IgM, IgG1, and IgG3 differentially associate with pro-inflammatory cytokines in HIV-infected men. Frontiers in Immunology 9 3141. (https://doi. org/10.3389/fimmu.2018.03141)

Potts WK, Manning CJ \& Wakeland EK 1991 Mating patterns in seminatural populations of mice influenced by MHC genotype. Nature 352 619-621. (https://doi.org/10.1038/352619a0)

Rametse CL, Adefuye AO, Olivier AJ, Curry L, Gamieldien H, Burgers WA, Lewis DA, Williamson AL, Katz AA \& Passmore JS 2018 Inflammatory cytokine profiles of semen influence cytokine responses of cervicovaginal epithelial cells. Frontiers in Immunology 9 2721. (https:// doi.org/10.3389/fimmu.2018.02721)

Robertson SA \& Sharkey DJ 2016 Seminal fluid and fertility in women. Fertility and Sterility 106 511-519. (https://doi.org/10.1016/j. fertnstert.2016.07.1101)
Robertson SA, Chin PY, Femia JG \& Brown HM 2018 Embryotoxic cytokines - potential roles in embryo loss and fetal programming. Journal of Reproductive Immunology 125 80-88. (https://doi.org/10.1016/j. jri.2017.12.003)

Rodgers AB, Morgan CP, Bronson SL, Revello S \& Bale TL 2013 Paternal stress exposure alters sperm microRNA content and reprograms offspring HPA stress axis regulation. Journal of Neuroscience 33 9003-9012. (https://doi.org/10.1523/JNEUROSCI.0914-13.2013)

Rodgers AB, Morgan CP, Leu NA \& Bale TL 2015 Transgenerational epigenetic programming via sperm microRNA recapitulates effects of paternal stress. PNAS 112 13699-13704. (https://doi.org/10.1073/ pnas.1508347112)

Sarkar P, Bergman K, O'Connor TG \& Glover V 2008 Maternal antenatal anxiety and amniotic fluid cortisol and testosterone: possible implications for fetal programming. Journal of Neuroendocrinology 20 489-496. (https://doi.org/10.1111/j.1365-2826.2008.01659.x)

Sarkey S, Azcoitia I, Garcia-Segura LM, Garcia-Ovejero D \& DonCarlos LL 2008 Classical androgen receptors in non-classical sites in the brain. Hormones and Behavior 53 753-764. (https://doi.org/10.1016/j. yhbeh.2008.02.015)

Schjenken JE \& Robertson SA 2015 Seminal fluid signalling in the female reproductive tract: implications for reproductive success and offspring health. Advances in Experimental Medicine and Biology 868 127-158. (https://doi.org/10.1007/978-3-319-18881-2_6)

Shirakata Y, Hiradate Y, Inoue H, Sato E \& Tanemura K 2014 Histone H4 modification during mouse spermatogenesis. Journal of Reproduction and Development 60 383-387. (https://doi.org/10.1262/ jrd.2014-018)

Stearns SC 1992 The Evolution of Life Histories, 1st ed. New York: Oxford University Press.

Tzanoulinou S, García-Mompó C, Castillo-Gómez E, Veenit V, Nacher J \& Sandi C 2014 Long-term behavioral programming induced by peripuberty stress in rats is accompanied by GABAergic-related alterations in the amygdala. PLOS ONE 9 e94666. (https://doi.org/10.1371/journal. pone.0094666)

Watanabe N \& Yamamoto M 2015 Neural mechanisms of social dominance. Frontiers in Neuroscience 9 154. (https://doi.org/10.3389/ fnins.2015.00154)

Weber EM, Algers B, Wurbel H, Hultgren J \& Olsson IAS 2013 Influence of strain and parity on the risk of litter loss in laboratory mice. Reproduction in Domestic Animals 48 292-296. (https://doi.org/10.1111/j.14390531.2012.02147.x)

Whitaker J, Moy SS, Godfrey V, Nielsen J, Bellinger D \& Bradfield J 2009 Effects of cage size and enrichment on reproductive performance and behavior in C57BL/6Tac mice. Lab Animal 38 24-34. (https://doi. org/10.1038/laban0109-24)

Zala SM, Potts WK \& Penn DJ 2004 Scent-marking displays provide honest signals of health and infection. Behavioral Ecology 15 338-344. (https:// doi.org/10.1093/beheco/arh022)

Zieminska E, Toczylowska B, Diamandakis D, Hilgier W, Filipkowski RK, Polowy R, Orzel J, Gorka M \& Lazarewicz JW 2018 Glutamate, glutamine and GABA levels in rat brain measured using MRS, HPLC and NMR methods in study of two models of autism. Frontiers in Molecular Neuroscience 11 418. (https://doi.org/10.3389/ fnmol.2018.00418)

Received 6 August 2019

First decision 12 September 2019

Revised manuscript received 19 April 2020

Accepted 24 April 2020 